\title{
An Improvement of Cardiovascular Risk Factors by Omega-3 Polyunsaturated Fatty Acids
}

\author{
Hidekatsu Yanai $^{\mathrm{a}, \mathrm{b}}$, Yoshinori Masui ${ }^{\mathrm{a}}$, Hisayuki Katsuyama ${ }^{\mathrm{a}}$, Hiroki Adachi ${ }^{\mathrm{a}}$, \\ Akiko Kawaguchi $^{\text {a }}$, Mariko Hakoshima ${ }^{\text {a }}$, Yoko Waragai ${ }^{\text {a }}$, \\ Tadanao Harigae ${ }^{a}$, Akahito Sako ${ }^{a}$
}

\begin{abstract}
An epidemiological survey in the Northwest Greenland reported that the Greenlanders have a lower frequency of acute myocardial infarction and diabetes mellitus. The very low incidence of ischemic heart disease in the Greenlanders was explained by consumption of a diet rich in omega-3 polyunsaturated fatty acids (PUFAs). Possible antiatherothrombotic effects of omega-3 PUFA include an improvement of lipid metabolism such as a reduction of triglyceride and an increase of high-density lipoprotein-cholesterol (HDL-C), and glucose metabolism, anti-platelet activity, anti-inflammatory effects, an improvement of endothelial function and stabilization of atherosclerotic plaque. The present study reviews an improvement of cardiovascular risk factors such as dyslipidemia and diabetes due to consumption of omega-3 PUFA. A sufficient number of studies suggest that omega-3 PUFA supplementation reduces serum triglyceride and increases HDL-cholesterol. The mechanisms for omega-3 PUFA-mediated improvements of lipid metabolism have been partially elucidated. The studies using experimental animals, part of trials in humans, have shown the beneficial effects of omega-3 PUFA on glucose metabolism and insulin sensitivity. The meta-analysis showed that omega-3 PUFA might prevent development of diabetes in part of population. Further studies should be performed to elucidate the association of omega-3 PUFA supplementation with diabetes, in the future.
\end{abstract}

Keywords: Diabetes; Insulin sensitivity; Omega-3 polyunsaturated fatty acids; Triglyceride

\section{Introduction}

An epidemiological survey of several chronic diseases in the

Manuscript submitted January 19, 2018, accepted February 7, 2018

aDepartment of Internal Medicine, National Center for Global Health and Medicine Kohnodai Hospital, Chiba, Japan

${ }^{\mathrm{b}}$ Corresponding Author: Hidekatsu Yanai, Department of Internal Medicine, National Center for Global Health and Medicine Kohnodai Hospital, 1-7-1 Kohnodai, Ichikawa, Chiba 272-8516, Japan.

Email: dyanai@hospk.ncgm.go.jp

doi: https://doi.org/10.14740/jocmr3362w
Upernavik district, Northwest Greenland, reported that the Greenlanders (Greenlandic Inuit) have a lower frequency of acute myocardial infarction (MI) and diabetes mellitus [1] (Fig. 1). To understand the etiology for the very low incidence of ischemic heart disease and diabetes, Bang et al studied plasma lipids and the composition of food consumed by the Greenlanders [2-4]. They found that the Greenlanders consumed a predominantly meat diet rich in omega-3 polyunsaturated fatty acids (PUFAs) such as eicosapentaenoic acid (EPA) and docosahexaenoic acid (DHA) [2] (Fig. 1). A recent prospective Danish cohort study also reported that incident MI was inversely related to a high intake of fatty fish [3]. Most types of lipids were decreased, compared with Danish people $[4,5]$. The most remarkable finding was a much lower level of plasma triglyceride (TG) in the Greenlanders than in Danish people $[4,5]$. Dyerberg et al found that the Greenlanders have high levels of EPA and low levels of arachidonic acid (AA) and they also have a tendency to bleed, suggesting that it is possible that dietary enrichment with EPA will protect against thrombosis [6]. The very low incidence of ischemic heart disease was explained by omega-3 PUFA-mediated reduction of TG-rich lipoproteins and anti-thrombotic effects.

Possible anti-atherothrombotic effects of omega-3 PUFA include an improvement of lipid metabolism such as a reduction of TG and an increase of high-density lipoprotein-cholesterol (HDL-C), and glucose metabolism, anti-platelet activity, anti-inflammatory effects, improvement of endothelial function and stabilization of atherosclerotic plaque [7].

In this review, we will think about an improvement of cardiovascular risk factors such as dyslipidemia and diabetes due to consumption of omega-3 PUFA.

\section{Materials and Methods}

We conducted a systematic review of studies of the association between omega-3 PUFA and coronary risk factors, by using MEDLINE/PubMed and Embase. The English-written literatures which reported a randomized controlled trial (RCT) were included, and non-English written literatures and/or literatures which reported observational studies were excluded. We also adopted literatures reporting RCT with appropriate statistical analysis. We considered that $\mathrm{P}<0.05$ was statistically significant. Eligible articles were found by using the combination of "fish", "fish oill", "sea food", "EPA", "DHA" and "omega-3 


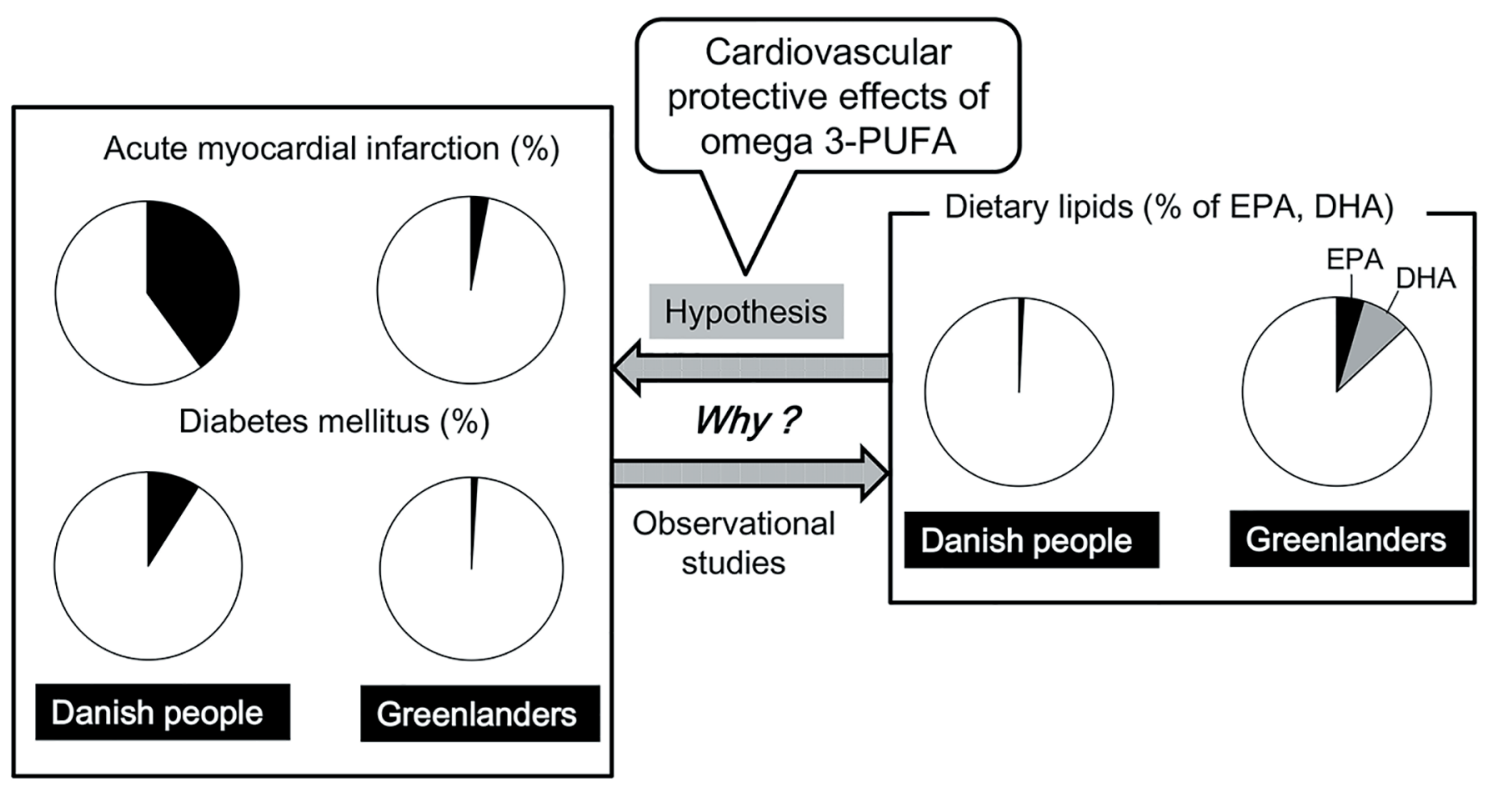

Figure 1. A lower prevalence of acute myocardial infarction and diabetes mellitus in the Greenlanders as compared with the Danish people, and the composition of food consumed by the Danish people and the Greenlanders. DHA: docosahexaenoic acid; EPA: eicosapentaenoic acid; PUFA: polyunsaturated fatty acids.

PUFA" with "lipid", "lipoprotein", "TG", "HDL", "LDL", "insulin sensitivity", "glucose", "insulin", "glycated hemoglobin", "HbA1c", and "diabetes" as the keywords. We partially used meta-analysis and systematic review including our previous studies $[8,9]$.

\section{Results}

\section{Effects on serum lipid metabolism}

\section{Effects in healthy, normolipidemic individuals}

The effects of fish diet, fish oil and DHA-rich oil on fasting and postprandial lipids in 55 healthy male students were investigated [10]. For 15 weeks, the subjects in the fish diet group ate $4.3 \pm 0.5$ fish containing meals $(0.38 \pm 0.04 \mathrm{~g}$ EPA and 0.67 $\pm 0.09 \mathrm{~g}$ DHA) per week and those in the fish oil (1.33 g EPA and $0.95 \mathrm{~g}$ DHA) and DHA-oil (1.68 $\mathrm{g}$ DHA) groups ate $4 \mathrm{~g}$ oil per day. The postprandial total and chylomicron (CM)-TG area under the curves (AUCs) were lowered in 15 weeks by the fish diet and fish oil $(\mathrm{P}<0.05)$, the same tendency $(\mathrm{P}<0.1)$ being seen in DHA-oil group. Forty-two normolipidemic adult subjects completed a double-blind placebo-controlled parallel study, receiving an EPA-rich oil (4.8 g EPA/day), DHA-rich oil (4.9 g DHA/day) or olive oil, for 4 weeks [11]. There was a significant $22 \%$ reduction in TG relative to the control following the DHA treatment $(\mathrm{P}=0.032)$, with the $15 \%$ decrease in the EPA group $(\mathrm{P}=0.258)$.

Seventy-four healthy normolipidemic men and women were randomly assigned to a total intake of $4.4 \mathrm{~g} /$ day alphalinolenic acid (ALA) (ALA group), $2.2 \mathrm{~g} /$ day EPA (EPA group), and $2.3 \mathrm{~g} /$ day DHA (DHA group) for 6 weeks [12]. Fasting serum TG was reduced in the EPA $(-0.14 \mathrm{mmol} / \mathrm{L})$ and DHA $(-0.30 \mathrm{mmol} / \mathrm{L})$ groups and also in the ALA group $(-0.17 \mathrm{mmol} / \mathrm{L})$. DHA intake significantly increased serum HDL-C, whereas no changes were found with ALA or EPA intake. Healthy men $(n=234)$ were randomly assigned to dietary supplementation with $3.8 \mathrm{~g} \mathrm{EPA} /$ day, $3.6 \mathrm{~g}$ DHA/day, or $4.0 \mathrm{~g}$ corn oil/day for 7 weeks [13]. Serum TG decreased 26\% $(\mathrm{P}<$ $0.0001)$ in the DHA group and $21 \%(\mathrm{P}=0.0001)$ in the EPA group compared with the corn oil group. Serum HDL-C increased to $0.06 \mathrm{mmol} / \mathrm{L}(\mathrm{P}=0.0002)$ in the DHA group, in the EPA group, serum total cholesterol decreased to $0.15 \mathrm{mmol} / \mathrm{L}$ $(\mathrm{P}=0.02)$ and apolipoprotein (apo) A-I decreased to $0.04 \mathrm{~g} / \mathrm{L}$ $(\mathrm{P}=0.0003)$.

Fourteen healthy volunteers were randomly allocated to receive daily $4 \mathrm{~g}$ EPA $(n=7)$ or DHA $(n=7)$ for 5 weeks with their ordinary diet [14]. The postprandial increase of TG was suppressed by $19 \%$ and $49 \%$ after prolonged intake of EPA and DHA, respectively. Healthy subjects $(n=33)$ were randomized to supplementation with $4 \mathrm{~g}$ /day of safflower oil, EPA, or DHA for 4 weeks [15]. Omega-3 PUFA supplementation reduced the postprandial TG and apoB-48 and apoB-100 concentrations by $16 \%(\mathrm{P}=0.08), 28 \%(\mathrm{P}<0.001)$, and $24 \%(\mathrm{P}<0.01)$, respectively. Omega-3 PUFA treatment reduced CM-TG half-lives. Omega-3 PUFA decreased CM particle sizes and increased pre-heparin lipoprotein lipase activity (LPL), suggesting that omega-3 PUFA supplementation accelerates CM-TG clearance by increasing LPL activity.

A 3-week daily intake of $3 \mathrm{~g}$ of EPA and DHA, a fish oil concentrate (FOC) (41\% EPA and 23\% DHA) and an olive oil were tested in 49 normolipidemic subjects [16]. FOC and EPA produced significant decreases in both TG and VLDL-C levels $(\mathrm{P}<0.01)$ and increases in LDL-C levels $(\mathrm{P}<0.05)$. DHA sup- 
plementation did not affect TG, VLDL, LDL, or HDL levels. Ten normo- and 10 dyslipidemic men were supplemented for 12 weeks with $1.14 \mathrm{~g}$ DHA and $1.56 \mathrm{~g}$ EPA [17]. Gene expression analyses revealed several possible molecular pathways by which EPA and DHA lower TG and increase HDL-C.

\section{Effects in obese and/or dyslipidemic individuals}

The effect of a 12-week omega-3 PUFA (46\% EPA and 38\% DHA) supplementation on apoB-48 kinetics in obese subjects on a weight-loss diet was examined [18]. Compared with weight loss alone, weight loss plus omega-3 PUFA significantly decreased fasting TG $(-11 \%)$, apoB-48 $(-36 \%)$, postprandial TG $(-21 \%)$, and apoB-48 (-22\%) total AUCs, as well as incremental postprandial TG AUCs (-32\%). The omega-3 PUFA significantly decreased apoB-48 secretion in the basal state, without a significant effect during the postprandial period, which suggests that omega-3 PUFAs improve CM metabolism by independently decreasing the secretion of apoB-48.

Twenty-four dyslipidemic, viscerally obese men were randomly assigned to receive either fish oil (4 g/day, 45\% EPA and 39\% DHA) or corn oil (4 g/day) for 6 weeks [19]. Fish oil supplementation significantly $(\mathrm{P}<0.05)$ lowered plasma $\mathrm{TG}$ $(-18 \%)$ and VLDL apoB $(-20 \%)$ and the hepatic secretion of VLDL apoB (-29\%) compared with placebo. Fish oils did not alter the catabolism of the $\mathrm{CM}$ remnants, suggesting that fish oils lower plasma TG, by decreasing VLDL apoB production but not by altering the catabolism of CM remnants.

Fifty-nine overweight, non-smoking, mildly hyperlipidemic men were randomly assigned to receive $4 \mathrm{~g}$ EPA, DHA, or olive oil daily while continuing their usual diets for 6 weeks [20]. Relative to those in the olive oil group, TG fell by approximately $20 \%(\mathrm{P}=0.003)$ in the DHA group and by approximately $18 \%(\mathrm{P}=0.012)$ in the EPA group. DHA increased LDL-C by $8 \%(\mathrm{P}=0.019)$. Adjusted LDL particle size increased with DHA but not with EPA.

Nozaki et al administered $2.7 \mathrm{~g}$ of EPA per day as capsules for 6 months to 14 primary hypercholesterolemic subjects [21]. Plasma TG and LDL-C were significantly reduced. Twenty male patients with primary hypertriglyceridemia were treated for 4 weeks with daily supplements $15 \mathrm{~g}$ of oil, which provided approximately $6 \mathrm{~g}$ of PUFA either of fish or of vegetable origin [22]. The fish, but not the vegetable, oil supplement led to a decrease in plasma TG. The decrease in plasma TG by fish-oil was accompanied by a reduction in the absolute catabolic rate of VLDL-TG, implying a concomitant change in synthetic rate; the fractional catabolic rate of VLDL-TG was unaltered.

A study comparing DHA to DHA + EPA in patients with coronary artery disease and TG greater than $200 \mathrm{mg} / \mathrm{dL}$ was conducted. Subjects were randomized to either $1,000 \mathrm{mg}$ of DHA or 1,252 mg of DHA + EPA for 8 weeks [23]. TG decreased by $21.8 \%$ in the DHA group $(\mathrm{P}<0.001)$ and $18.3 \%$ in the DHA + EPA group $(\mathrm{P}<0.001)$. The difference between groups was not significant. Hypertriglyceridemic subjects were randomly assigned to a control group $(\mathrm{n}=72)$ or an EPA group ( $\mathrm{n}=72 ; \mathrm{EPA} 1,800 \mathrm{mg} /$ day for 6 months) [24]. In the EPA group, the serum LDL-C and HDL-C levels remained un-
Table 1. Comparison of Effects of EPA and DHA on Serum Lipids

\begin{tabular}{lll} 
& EPA & DHA \\
\hline Triglyceride & $\downarrow$ & $\downarrow \downarrow$ \\
HDL-cholesterol & $\uparrow$ & $\uparrow \uparrow$ \\
LDL-cholesterol & $\downarrow$ & $\uparrow$ \\
LDL particle size & & $\uparrow$ \\
\hline
\end{tabular}

changed, but there was a significant increase in LDL particle size, and a significant decrease in serum TG-rich lipoproteins.

In the multicenter, 12-week, double-blind study, subjects were stratified for co-administration of a 3-hydroxy-3-methylglutaryl-CoA reductase inhibitor then randomized to EPA + DHA 2 g once daily $(\mathrm{n}=205), \mathrm{EPA}+$ DHA $2 \mathrm{~g}$ twice daily ( $=210)$, or EPA $0.6 \mathrm{~g}$ three times daily $(\mathrm{n}=195)$ [25]. Each one gram of fatty acid in EPA + DHA contains approximately 465 $\mathrm{mg}$ of EPA plus $375 \mathrm{mg}$ of DHA. EPA + DHA $4 \mathrm{~g} /$ day reduced TG more than EPA $1.8 \mathrm{~g} /$ day. EPA + DHA $2 \mathrm{~g} /$ day had similar effects on TG as EPA $1.8 \mathrm{~g}$ /day.

\section{Effects in individuals with type 2 diabetes}

The effect of a daily intake of $1,080 \mathrm{mg}$ EPA and $720 \mathrm{mg}$ DHA (8 weeks) for diabetic subjects on the kinetics of apoB100 -containing lipoprotein in the fasting state was determined [26]. Treatment using EPA and DHA lowered serum TG from $1.776 \pm 0.280$ to $1.356 \pm 0.595 \mathrm{mmol} / \mathrm{L}(\mathrm{P}<0.05)$. A decrease in VLDL apoB-100 concentration from $0.366 \pm 0.030$ to 0.174 $\pm 0.036 \mathrm{~g} / \mathrm{L}(\mathrm{P}<0.05)$, with no change in fractional catabolic rates, was observed.

In an RCT, 59 subjects with type 2 diabetes were randomly assigned to consume $4 \mathrm{~g}$ EPA, DHA, or olive oil/day for 6 weeks while continuing to consume their usual diet [27]. Serum TG in the EPA and DHA groups decreased $19 \%(\mathrm{P}=$ $0.022)$ and $15 \%(\mathrm{P}=0.022)$, respectively. There were no significant changes in serum LDL-C, or HDL-C.

\section{Effects on other atherogenic lipoproteins}

Twenty-two hemodialysis and 16 continuous ambulatory peritoneal dialysis patients with relatively high plasma levels of remnant lipoproteins and oxidized-LDL were randomized to either EPA or placebo [28]. Highly purified EPA was administered at a dose of $1,800 \mathrm{mg}$ daily for 3 months. EPA significantly reduced the levels of both remnant lipoproteins $(52 \%$ reduction) and ox-LDL (38\% reduction).

\section{EPA vs. DHA}

Comparison of effects of EPA and DHA on TG, HDL-C, LDL$\mathrm{C}$ and LDL particle size is shown in Table 1 . Wei et al performed a meta-analysis of RCTs of monotherapy with EPA ( $\mathrm{n}$ $=10)$, DHA $(n=17)$, or EPA versus DHA $(n=6)$ [29]. Com- 
pared with placebo, DHA raised LDL $7.23 \mathrm{mg} / \mathrm{dL}$ (95\% CI, 3.98 - 10.5) whereas EPA non-significantly reduced LDL. In direct comparison studies, DHA raised LDL $4.63 \mathrm{mg} / \mathrm{dL}(95 \%$ CI, 2.15 - 7.10) more than EPA. Both EPA and DHA reduced $\mathrm{TG}$, with a greater reduction by DHA in direct comparison studies. DHA also raised HDL (4.49 mg/dL; 95\% CI, 3.50 5.48) compared with placebo, whereas EPA did not.

Placebo-adjusted changes in mean lipid parameters were compared in RCTs in subjects treated for $\geq 4$ weeks with DHA or EPA [30]. Twenty-two studies were identified. A net increase in LDL-C of 3.3\% was observed with DHA (DHA: $+2.6 \%$; EPA: $-0.7 \%$ ). DHA treatment was associated with a net decrease in TG by $6.8 \%$ (DHA: $-22.4 \%$; EPA: $-15.6 \%$ ), and a net increase in HDL-C by $5.9 \%$ (DHA: $+7.3 \%$; EPA: $+1.4 \%$ ). Increases in LDL-C were also observed in $71 \%$ of DHA-alone groups, but not in any EPA-alone studies. In another systematic review, only DHA decreased the number of small dense LDL particles [31].

\section{Effects on glucose metabolism and the development of dia- betes}

\section{Effects on glucose metabolism and insulin sensitivity}

One hundred sixty-two healthy individuals were randomly assigned to diets rich in monounsaturated fats and the other rich in saturated fats for 3 months. Within each group, there was a second randomization to fish oil (omega-3 PUFA $3.6 \mathrm{~g} /$ day) or placebo for 3 months. A moderate supplementation of fish oil did not affect insulin sensitivity, insulin secretion, beta-cell function or glucose tolerance [32]. In a randomized, parallel design in 258 subjects aged 45 - 70 years old, four diets providing $6 \%$ of energy as PUFA with an $(n-6) /(n-3)$ between $5: 1$ and $3: 1$ with a control diet that had an $(n-6) /(n-3)$ of $10: 1$ were compared [33]. Changes in the $(n-6) /(n-3)$ did not influence insulin sensitivity.

In an RCT, Stirban et al investigated the effects of EPA/ DHA on paraoxonase-1 activity as well as fasting and postprandial levels of circulating adiponectin and leptin in 34 subjects with type 2 diabetes who received daily for 6 weeks either $2 \mathrm{~g}$ purified EPA/DHA or olive oil, separated by a 6 weeks washout. No significant differences in fasting and postprandial circulating adiponectin, leptin, and paraoxonase-1 activity were seen between omega-3 PUFAs and placebo [34].

Thirty-one insulin-resistant adults received $3.9 \mathrm{~g} /$ day EPA + DHA or placebo for 6 months in a randomized double-blind study. Compared with placebo, EPA + DHA did not alter peripheral insulin sensitivity, postprandial glucose disposal, or insulin secretion. Hepatic insulin sensitivity exhibited a small but significant improvement with EPA + DHA compared with placebo [35].

Sixty-four patients, who were hypertensive and/or diabetic obese with high levels of inflammatory markers, from primary healthcare centers of Gaza City, Palestine, were enrolled in two groups of an open-label, parallel, randomized, controlled trial for 8 weeks [36]. Thirty-three patients were in the control group, and 31 patients were in the experimental group. The ex- perimental group was treated with a daily dose of $300 \mathrm{mg}$ EPA and $200 \mathrm{mg}$ of DHA. Treatment with omega-3 PUFA significantly reduced the level of high-sensitivity $\mathrm{C}$-reactive protein, fasting blood glucose, and TG after 8 weeks of treatment.

Type 2 diabetic patients $(n=38)$ treated with oral hypoglycemic agents, without insulin were supplemented with omega-3 PUFA (1.2 g/day) for 2 months [37]. Omega-3 PUFA supplementation significantly reduced fasting glucose, glycated hemoglobin levels.

In an RCT, 100 type 2 diabetic patients with abdominal obesity were randomized into two groups including $4 \mathrm{~g}$ /day of fish oil (2.4 g omega-3 PUFA) or corn oil for 6 months [38]. Serum TG decreased $(\mathrm{P}=0.007)$, whereas HDL-C increased $(\mathrm{P}=0.006)$ in the fish oil group compared with the placebo group after 6 months. Glycemic control which was measured by serum glucose, glycated hemoglobin, insulin, and homeostasis model assessment-insulin resistance, was not significantly different between the two groups after 6 months. In an RCT, 54 patients with type 2 diabetes received $520 \mathrm{mg}$ of DHA + EPA-enriched fish-oil (FOG) or a placebo daily [39]. Waist circumference and blood glucose showed significant reductions in the FOG group $(\mathrm{P}=0.001$ and $\mathrm{P}=0.011$, respectively).

The RCT included 43 patients with type 2 diabetes $(21$ patients in the placebo group and 22 patients in the omega- 3 PUFA group) [40]. They were randomized to groups, one receiving 10 weeks of either omega-3 PUFA supplement and the other the placebo (1,250 $\mathrm{mg}$ capsule, three times per day). Compared to the placebo, omega-3 PUFA supplementation decreased serum fasting plasma glucose and glycated hemoglobin $(\mathrm{P}=0.036$ and 0.001 , respectively).

Akinkuolie et al systematically reviewed the effect of n-3 PUFA on insulin sensitivity by conducting a meta-analysis of available RCTs [41]. Eleven RCTs $(n=618)$ were eligible for inclusion in the analysis. In a pooled estimate, omega-3 PUFA intervention had no effects on insulin sensitivity compared to placebo.

To determine whether omega-3 PUFA interventions affect insulin resistance in a sex-dependent manner, five databases were searched (Medline, EMBASE, CINAHL, Scopus, and Pre-Medline) for RCTs [42]. With all studies $(n=26)$ pooled, there was no effect of omega-3 PUFA on insulin resistance at the group level. In trials with duration $\geq 6$ weeks, a significant improvement in insulin resistance was seen in women but not in men.

Literature searches of Medline, PubMed Central and Embase were conducted up to December 2015 [43]. All RCTs that investigated effects of omega-3 PUFA supplements on insulin resistance in women with polycystic ovary syndrome (PCOS) were included. Of 1,202 papers, three RCTs were eligible for inclusion which involved 72 cases and 73 controls. The dose range for omega-3 PUFA supplement was 1.2 to $3.6 \mathrm{~g}$ and the duration of follow-up was from 6 to 8 weeks. There was no significant effect of omega-3 PUFA supplements compared to placebo on insulin resistance in women with PCOS.

To systematically evaluate the effects of omega-3 PUFA on glucose control and lipid levels, Medline, PubMed, Cochrane Library, Embase, the National Research Register, and SIGLE were searched to identify eligible RCTs [44]. Twenty RCTs were included into this meta-analysis. Among patients with 
omega-3 PUFA supplementation, no marked change in glycated hemoglobin, fasting plasma glucose, and postprandial plasma glucose was observed.

To understand whether the net effect of fish oil intake on glycemic control is beneficial in diabetes, a meta-analysis was performed [45]. Data sources were Medline (Cologne, Germany), Excerpta Medica, Current Contents, review articles, and published reference lists. Publications of 26 trials were selected, and all trials included more than five diabetes patients and addressed the effects of fish oil (EPA and DHA) on serum lipids and glucose tolerance. No significant change in glycated hemoglobin occurred in diabetic subjects treated with fish oil.

\section{Effects on the development of diabetes}

We previously reported a systematic review on the effects of fish or fish oils on the development of diabetes [8]. Wallin et al performed a systematic review and meta-analysis to understand the association between fish consumption, dietary omega-3 PUFA, and risk of type 2 diabetes, by searching the PubMed and Embase databases through December 15, 2011 [46]. Sixteen studies involving 527,441 participants and 24,082 diabetes cases were included. For each serving per week increment in fish consumption, the relative risk (RR) (95\% confidence interval (CI)) of type 2 diabetes was 1.05 (1.02 - 1.09), 1.03 (0.96 - 1.11), and 0.98 (0.97 - 1.00), in combined analysis of studies performed in US, European, and Asian/Australian, respectively. For each $0.30 \mathrm{~g}$ per day increment in long-chain omega-3 PUFA, the corresponding summary estimates were 1.17 (1.09 - 1.26), 0.98 (0.70 - 1.37), and 0.90 (0.82 - 0.98). This meta-analysis indicated differences between geographical regions in observed associations of fish consumption and dietary intake of omega-3 PUFA with risk of type 2 diabetes.

Zheng et al conducted a systematic review and meta-analysis of prospective cohort studies to examine the associations of fish and omega-3 PUFA intake with type 2 diabetes risk [47]. Twenty-four studies including 24,509 type 2 diabetic patients and 545,275 participants were identified. For cohort studies, the summary RR of type 2 diabetes for the highest vs. lowest categories of total fish and omega-3 PUFA intake was 1.07 (95\% CI: 0.91 - 1.25) and 1.07 (95\% CI: 0.95 - 1.20), respectively. Subgroup analyses indicated that summary RR (highest vs. lowest category) of type 2 diabetes for fish and omega-3 PUFA intake was 0.89 (95\% CI: $0.81-0.98)$ and 0.87 (95\% CI: $0.79-0.96)$ for Asian populations, and $1.20(95 \%$ CI: 1.01 - 1.44) and 1.16 (95\% CI: 1.04 - 1.28) for Western populations. This systematic review and meta-analysis showed that omega-3 PUFA have beneficial effects on the prevention of type 2 diabetes in Asian populations.

$\mathrm{Wu}$ et al systematically searched multiple literature databases through June 2011 to identify prospective studies examining relations of dietary omega-3 PUFA, dietary fish and/or seafood, and circulating omega-3 PUFA biomarkers with incidence of diabetes [48]. Sixteen studies met inclusion criteria, including 18 separate cohorts comprising 540,184 individuals and 25,670 cases of incident diabetes. Consumption of fish and/or seafood was not significantly associated with diabetes, nor was consumption of EPA + DHA.
There is a considerable statistical heterogeneity in the overall summary estimates of the association between fish consumption, dietary omega-3 PUFA, and risk of type 2 diabetes, which is partly explained by geographical differences. Omega-3 PUFAs have beneficial effects on the prevention of type 2 diabetes in Asian populations.

\section{Discussion}

A sufficient number of RCTs showed omega-3 PUFA reduced serum TG. The mechanisms for omega-3 PUFA-mediated TG reduction remain largely unknown. Omega-3 PUFA reduced the postprandial CM-TG AUCs [10], and reduced apoB-48 which is the apolipoprotein of CM [15]. Omega-3 PUFA also decreased CM particle sizes and increased pre-heparin LPL, suggesting that omega-3 PUFA supplementation accelerates CM-TG clearance by increasing LPL activity [15]. Intestinal $\mathrm{CM}$ production is upregulated in humans under conditions of insulin resistance [49]. CM remnants possess direct atherogenic properties [49]. The omega-3 PUFA was reported to significantly decreased apoB-48 secretion in the basal state [18]. Reduced secretion of apoB-48 may reduce production of $\mathrm{CM}$, and may also induce a decrease of CM remnants which is beneficial to prevent atherosclerosis. A significant reduction of remnant lipoproteins (52\% reduction) due to EPA supplementation was observed, supporting our hypothesis [28]. Briefly, omega-3 PUFA reduces atherogenic CM remnant by increase of LPL activity and reduction of apoB-48 secretion.

Omega-3 PUFA significantly produced a significant decrease in VLDL-C and hepatic secretion of VLDL apoB as compared with placebo $[19,26]$. Omega-3 PUFA was also reported to reduce the postprandial apoB-100 concentration by $24 \%$ [13]. The reduction of hepatic secretion of apoB-100 which is apolipoprotein of VLDL may produce a significant reduction of VLDL production.

Omega-3 PUFAs elicit hypotriglyceridemic effects by suppressing hepatic lipogenesis through reducing levels of sterol receptor element binding protein-1c (SREBP-1c), upregulating FA oxidation in the liver and skeletal muscle [50]. The net result is the repartitioning of metabolic fuel from TG storage toward oxidation, thereby reducing the substrate available for VLDL synthesis.

Omega-3 PUFAs, especially DHA, increase HDL-C [12, $13,29,30]$. The formation of HDL is related with the catabolism of TG-rich lipoproteins such as VLDL or intermediatedensity lipoprotein (IDL) by LPL [51]. Therefore, increased LPL activity reduces IDL and VLDL, and increases HDL. Increased pre-heparin LPL due to omega-3 PUFA was observed [15]. An increase of HDL-C due to omega-3 PUFA can be explained by an increased activity of LPL, which may be associated with increased TG hydrolysis of VLDL. Omega-3 PUFA reduces VLDL-C, by reduction of hepatic secretion of apoB100, decrease of SREBP-1c, and an increase of LPL activity.

DHA raised LDL $7.23 \mathrm{mg} / \mathrm{dL}$ (95\% CI, 3.98 - 10.5), whereas EPA non-significantly reduced LDL [29]. Increases in LDL-C were observed in $71 \%$ of DHA-alone groups, but not in any EPA-alone studies [30]. Can this elevation of LDL- 


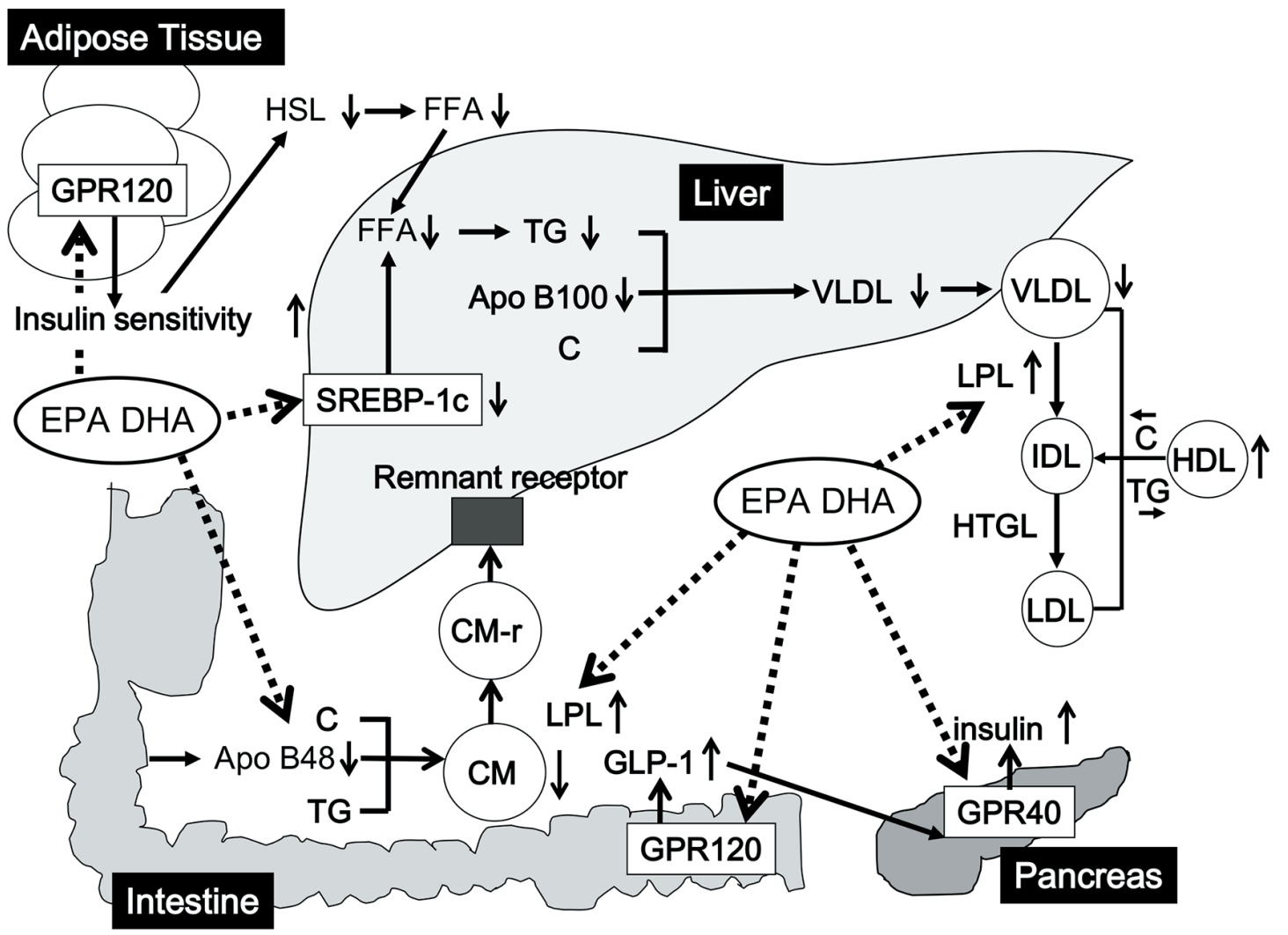

Figure 2. The possible mechanisms for omega-3 PUFA-mediated improvements in lipid and glucose metabolism. Dash lines indicate direct effects of EPA and DHA, and solid lines indicate secondary changes of metabolic state in lipid/glucose metabolism due to EPA and DHA. C: cholesterol; CM: chylomicron; CM-r: chylomicron remnant; DHA: docosahexaenoic acid; EPA: eicosapentaenoic acid; FFA: free fatty acids; GLP-1: glucagon-like peptide 1; GPR: G protein-coupled receptor; HSL: hormonesensitive lipase; HTGL: hepatic triglyceride lipase; IDL: intermediate-density lipoprotein (IDL); LDL: low-density lipoprotein; LPL: lipoprotein lipase; PUFA: polyunsaturated fatty acids; SREBP: sterol receptor element binding protein; TG: triglyceride; VLDL: very low-density lipoprotein.

C induce atherosclerosis? We think that the answer is "No". DHA reduces more VLDL than EPA. Therefore, DHA increases more LDL which is the TG-hydrolyzed product of VLDL as compared with EPA. Further, the particle size of LDL elevated by DHA is large [20], and a large-sized LDL may not induce atherosclerosis. The systematic review which showed DHA decreased the number of small dense LDL particles [31] supports our hypothesis.

The effects of long chain FA in pancreatic beta-cells are mediated by a group of G-protein coupled receptors [52]. The G protein-coupled receptor 40 (GPR40) appears to mediate the acute stimulatory effects of long chain FA on insulin secretion. Stimulation of GPR120 with omega-3 PUFA causes broad anti-inflammatory effects in macrophages [53]. GPR120 is a functional omega-3 PUFA receptor/sensor and mediates potent insulin sensitizing and antidiabetic effects in vivo. Further, an improvement of insulin sensitivity suppresses the activity of hormone-sensitive lipase (HSL) in adipose tissue which catalyzes the breakdown of TG, releasing free FA [54].

After an intraperitoneal glucose challenge, marked endogenous GLP-1 secretion, substantial insulin release and subsequent glucose reductions were observed in the intracolonic
DHA and EPA treatment mice, suggesting that the colon-specific delivery of omega-3 PUFA would be a novel antidiabetic treatment by the stimulation of intrinsic GLP-1 secretion via GPR120 [55].

I previously presented the review compared between the effect to prevent cardiovascular events by only EPA and the effect by the combination of EPA and DHA [56]. Statins are the "gold standard" for the treatment to prevent cardiovascular diseases. To evaluate the cardio-protective effects of some drugs in clinical trials, we should consider whether trial participants take statins or not. In Japan, EPA lipid intervention study (JELIS), all participants had taken statins, and a 19\% relative reduction in major coronary events was obtained by the addition EPA [57].

In the GISSI-Prevenzione (GISSI-P) trial, the treatment with n-3 FA (EPA + DHA) significantly lowered the risk of the primary endpoint (RR decrease 15\% (95\% CI, 2 - 26)) [58]. In the GISSI-HF trial, the adjusted hazard ratio (HR) of death from any cause in the n-3 PUFA (EPA + DHA) group was 0.91 (95.5\% CI: $0.833-0.998), \mathrm{P}=0.041$ ) [59]. The adjusted HR of death or admission to hospital for cardiovascular reasons was 0.92 (99\% CI: $0.849-0.999), \mathrm{P}=0.009)$. The percentages of 
subjects who had taken satins were only $4.7 \%$ and $22.6 \%$ in GISSI-P and GISSI-HF trials, respectively.

The recent OMEGA study including 94.2\% statin users failed to prevent cardiovascular events [60]. The ORIGIN trial including 53.8\% statin users also failed [61]. In the previous two studies (GISSI-P and GISSI-HF), EPA + DHA may be effective to prevent cardiovascular events because of fewer combined use of statins. In the recent studies (OMEGA and ORIGIN) which included many participants who had taken statins, EPA + DHA failed to reduce cardiovascular events. However, EPA demonstrated a significant reduction of cardiovascular events in JELIS, in which all participants had taken statins. We should also consider how EPA or DHA reduces cardiovascular events independently of an improvement of lipid metabolism. EPA or DHA may reduce cardiovascular events, due to decrease of inflammation and/or platelets activity, or the improvement of vascular integrity.

\section{Conclusion}

The possible mechanisms for omega-3 PUFA-mediated improvements in lipid and glucose metabolism were shown in Figure 2. Omega-3 PUFA reduces atherogenic CM remnant by increase of LPL activity and reduction of apoB-48 secretion, and reduces VLDL-C, by reduction of hepatic secretion of apoB-100, decrease of SREBP-1c, and an increase of LPL activity. Omega-3 PUFA raises HDL by increase of LPL activity. Omega-3 PUFA increases insulin secretion from pancreatic beta-cells by stimulating GPR40, and increases GLP-1 and improves insulin sensitivity by stimulating GPR120.

\section{Competing Interests}

The authors declare that they have no competing interests concerning this article.

\section{References}

1. Kromann N, Green A. Epidemiological studies in the Upernavik district, Greenland. Incidence of some chronic diseases 1950-1974. Acta Med Scand. 1980;208(5):401406.

2. Bang HO, Dyerberg J, Hjoorne N. The composition of food consumed by Greenland Eskimos. Acta Med Scand. 1976;200(1-2):69-73.

3. Gammelmark A, Nielsen MS, Bork CS, Lundbye-Christensen S, Tjonneland A, Overvad K, Schmidt EB. Association of fish consumption and dietary intake of marine n-3 PUFA with myocardial infarction in a prospective Danish cohort study. Br J Nutr. 2016;116(1):167-177.

4. Bang HO, Dyerberg J. Plasma lipids and lipoproteins in Greenlandic west coast Eskimos. Acta Med Scand. 1972;192(1-2):85-94.

5. Bang HO, Dyerberg J, Nielsen AB. Plasma lipid and lipoprotein pattern in Greenlandic West-coast Eskimos. Lan- cet. $1971 ; 1(7710): 1143-1145$.

6. Dyerberg J, Bang HO, Stoffersen E, Moncada S, Vane JR. Eicosapentaenoic acid and prevention of thrombosis and atherosclerosis? Lancet. 1978;2(8081):117-119.

7. Yanai H, Yoshida H. Eicosapentaenoic Acids (EPA) for Atherosclerotic Diseases. JSMUFF. 2014;8(3):215-220.

8. Yanai H, Hamasaki H, Katsuyama H, Adachi H, Moriyama S, Sako A. Effects of intake of fish or fish oils on the development of diabetes. J Clin Med Res. 2015;7(1):812.

9. Yanai H, Katsuyama H, Hamasaki H, Abe S, Tada N, Sako A. Effects of dietary fat intake on HDL metabolism. J Clin Med Res. 2015;7(3):145-149.

10. Agren JJ, Hanninen O, Julkunen A, Fogelholm L, Vidgren $\mathrm{H}$, Schwab U, Pynnonen O, et al. Fish diet, fish oil and docosahexaenoic acid rich oil lower fasting and postprandial plasma lipid levels. Eur J Clin Nutr. 1996;50(11):765-771.

11. Buckley R, Shewring B, Turner R, Yaqoob P, Minihane AM. Circulating triacylglycerol and apoE levels in response to EPA and docosahexaenoic acid supplementation in adult human subjects. Br J Nutr. 2004;92(3):477483.

12. Egert S, Kannenberg F, Somoza V, Erbersdobler HF, Wahrburg U. Dietary alpha-linolenic acid, EPA, and DHA have differential effects on LDL fatty acid composition but similar effects on serum lipid profiles in normolipidemic humans. J Nutr. 2009;139(5):861-868.

13. Grimsgaard S, Bonaa KH, Hansen JB, Nordoy A. Highly purified eicosapentaenoic acid and docosahexaenoic acid in humans have similar triacylglycerol-lowering effects but divergent effects on serum fatty acids. Am J Clin Nutr. 1997;66(3):649-659.

14. Hansen JB, Grimsgaard S, Nilsen H, Nordoy A, Bonaa KH. Effects of highly purified eicosapentaenoic acid and docosahexaenoic acid on fatty acid absorption, incorporation into serum phospholipids and postprandial triglyceridemia. Lipids. 1998;33(2):131-138.

15. Park Y, Harris WS. Omega-3 fatty acid supplementation accelerates chylomicron triglyceride clearance. J Lipid Res. 2003;44(3):455-463.

16. Rambjor GS, Walen AI, Windsor SL, Harris WS. Eicosapentaenoic acid is primarily responsible for hypotriglyceridemic effect of fish oil in humans. Lipids. 1996;31(Suppl):S45-49.

17. Schmidt S, Willers J, Stahl F, Mutz KO, Scheper T, Hahn A, Schuchardt JP. Regulation of lipid metabolism-related gene expression in whole blood cells of normo- and dyslipidemic men after fish oil supplementation. Lipids Health Dis. 2012;11:172.

18. Wong AT, Chan DC, Barrett PH, Adams LA, Watts GF. Effect of omega-3 fatty acid ethyl esters on apolipoprotein B-48 kinetics in obese subjects on a weight-loss diet: a new tracer kinetic study in the postprandial state. J Clin Endocrinol Metab. 2014;99(8):E1427-1435.

19. Chan DC, Watts GF, Mori TA, Barrett PH, Redgrave TG, Beilin LJ. Randomized controlled trial of the effect of $n-3$ fatty acid supplementation on the metabolism of apolipoprotein B-100 and chylomicron remnants in men with 
visceral obesity. Am J Clin Nutr. 2003;77(2):300-307.

20. Mori TA, Burke V, Puddey IB, Watts GF, O’Neal DN, Best JD, Beilin LJ. Purified eicosapentaenoic and docosahexaenoic acids have differential effects on serum lipids and lipoproteins, LDL particle size, glucose, and insulin in mildly hyperlipidemic men. Am J Clin Nutr. 2000;71(5):1085-1094.

21. Nozaki S, Matsuzawa Y, Hirano K, Sakai N, Kubo M, Tarui S. Effects of purified eicosapentaenoic acid ethyl ester on plasma lipoproteins in primary hypercholesterolemia. Int J Vitam Nutr Res. 1992;62(3):256-260.

22. Sanders TA, Sullivan DR, Reeve J, Thompson GR. Triglyceride-lowering effect of marine polyunsaturates in patients with hypertriglyceridemia. Arteriosclerosis. 1985;5(5):459-465.

23. Schwellenbach LJ, Olson KL, McConnell KJ, Stolcpart RS, Nash JD, Merenich JA. The triglyceride-lowering effects of a modest dose of docosahexaenoic acid alone versus in combination with low dose eicosapentaenoic acid in patients with coronary artery disease and elevated triglycerides. J Am Coll Nutr. 2006;25(6):480-485.

24. Tani S, Nagao K, Matsumoto M, Hirayama A. Highly purified eicosapentaenoic acid may increase low-density lipoprotein particle size by improving triglyceride metabolism in patients with hypertriglyceridemia. Circ J. 2013;77(9):2349-2357.

25. Tatsuno I, Saito Y, Kudou K, Ootake J. Efficacy and safety of TAK-085 compared with eicosapentaenoic acid in Japanese subjects with hypertriglyceridemia undergoing lifestyle modification: the omega-3 fatty acids randomized double-blind (ORD) study. J Clin Lipidol. 2013;7(3):199-207.

26. Ouguerram K, Maugeais C, Gardette J, Magot T, Krempf M. Effect of n-3 fatty acids on metabolism of apoB100containing lipoprotein in type 2 diabetic subjects. Br J Nutr. 2006;96(1):100-106.

27. Woodman RJ, Mori TA, Burke V, Puddey IB, Watts GF, Beilin LJ. Effects of purified eicosapentaenoic and docosahexaenoic acids on glycemic control, blood pressure, and serum lipids in type 2 diabetic patients with treated hypertension. Am J Clin Nutr. 2002;76(5):1007-1015.

28. Ando M, Sanaka T, Nihei H. Eicosapentanoic acid reduces plasma levels of remnant lipoproteins and prevents in vivo peroxidation of LDL in dialysis patients. J Am Soc Nephrol. 1999;10(10):2177-2184.

29. Wei MY, Jacobson TA. Effects of eicosapentaenoic acid versus docosahexaenoic acid on serum lipids: a systematic review and meta-analysis. Curr Atheroscler Rep. 2011;13(6):474-483.

30. Jacobson TA, Glickstein SB, Rowe JD, Soni PN. Effects of eicosapentaenoic acid and docosahexaenoic acid on low-density lipoprotein cholesterol and other lipids: a review. J Clin Lipidol. 2012;6(1):5-18.

31. Kelley DS, Adkins Y. Similarities and differences between the effects of EPA and DHA on markers of atherosclerosis in human subjects. Proc Nutr Soc. 2012;71(2):322-331.

32. Giacco R, Cuomo V, Vessby B, Uusitupa M, Hermansen K, Meyer BJ, Riccardi G, et al. Fish oil, insulin sensitivity, insulin secretion and glucose tolerance in healthy peo- ple: is there any effect of fish oil supplementation in relation to the type of background diet and habitual dietary intake of n-6 and n-3 fatty acids? Nutr Metab Cardiovasc Dis. 2007; 17(8):572-580.

33. Griffin MD, Sanders TA, Davies IG, Morgan LM, Millward DJ, Lewis F, Slaughter S, et al. Effects of altering the ratio of dietary n-6 to n-3 fatty acids on insulin sensitivity, lipoprotein size, and postprandial lipemia in men and postmenopausal women aged 45-70 y: the OPTILIP Study. Am J Clin Nutr. 2006;84(6):1290-1298.

34. Stirban A, Nandrean S, Gotting C, Stratmann B, Tschoepe D. Effects of $n-3$ polyunsaturated fatty acids (PUFAs) on circulating adiponectin and leptin in subjects with type 2 diabetes mellitus. Horm Metab Res. 2014;46(7):490-492.

35. Lalia AZ, Johnson ML, Jensen MD, Hames KC, Port JD, Lanza IR. Effects of dietary n-3 fatty acids on hepatic and peripheral insulin sensitivity in insulin-resistant humans. Diabetes Care. 2015;38(7):1228-1237.

36. Ellulu MS, Khaza'ai H, Patimah I, Rahmat A, Abed Y. Effect of long chain omega-3 polyunsaturated fatty acids on inflammation and metabolic markers in hypertensive and/ or diabetic obese adults: a randomized controlled trial. Food Nutr Res. 2016;60:29268.

37. Kurt A, Andican G, Siva ZO, Andican A, Burcak G. The effects of n-3 long-chain polyunsaturated fatty acid supplementation on AGEs and sRAGE in type 2 diabetes mellitus. J Physiol Biochem. 2016;72(4):679-687.

38. Wang F, Wang Y, Zhu Y, Liu X, Xia H, Yang X, Sun G. Treatment for 6 months with fish oil-derived n-3 polyunsaturated fatty acids has neutral effects on glycemic control but improves dyslipidemia in type 2 diabetic patients with abdominal obesity: a randomized, double-blind, placebo-controlled trial. Eur J Nutr. 2017;56(7):24152422.

39. Jacobo-Cejudo MG, Valdes-Ramos R, GuadarramaLopez AL, Pardo-Morales RV, Martinez-Carrillo BE, Harbige LS. Effect of n-3 polyunsaturated fatty acid supplementation on metabolic and inflammatory biomarkers in type 2 diabetes mellitus patients. Nutrients. 2017;9(6):573.

40. Ansari S, Djalali M, Mohammadzadeh Honarvar N, Mazaherioun M, Zarei M, Agh F, Gholampour Z, et al. The effect of n-3 polyunsaturated fatty acids supplementation on serum irisin in patients with type 2 diabetes: a randomized, double-blind, placebo-controlled trial. Int J Endocrinol Metab. 2017;15(1):e40614.

41. Akinkuolie AO, Ngwa JS, Meigs JB, Djousse L. Omega-3 polyunsaturated fatty acid and insulin sensitivity: a meta-analysis of randomized controlled trials. Clin Nutr. 2011;30(6):702-707.

42. Abbott KA, Burrows TL, Thota RN, Acharya S, Garg ML. Do omega-3 PUFAs affect insulin resistance in a sex-specific manner? A systematic review and metaanalysis of randomized controlled trials. Am J Clin Nutr. 2016;104(5):1470-1484.

43. Sadeghi A, Djafarian K, Mohammadi H, Shab-Bidar S. Effect of omega-3 fatty acids supplementation on insulin resistance in women with polycystic ovary syndrome: Meta-analysis of randomized controlled trials. Diabetes 
Metab Syndr. 2017;11(2):157-162.

44. Chen C, Yu X, Shao S. Effects of omega-3 fatty acid supplementation on glucose control and lipid levels in type 2 diabetes: a meta-analysis. PLoS One. 2015;10(10):e0139565.

45. Friedberg CE, Janssen MJ, Heine RJ, Grobbee DE. Fish oil and glycemic control in diabetes. A meta-analysis. Diabetes Care. 1998;21(4):494-500.

46. Wallin A, Di Giuseppe D, Orsini N, Patel PS, Forouhi NG, Wolk A. Fish consumption, dietary long-chain n-3 fatty acids, and risk of type 2 diabetes: systematic review and meta-analysis of prospective studies. Diabetes Care. 2012;35(4):918-929.

47. Zheng JS, Huang T, Yang J, Fu YQ, Li D. Marine N-3 polyunsaturated fatty acids are inversely associated with risk of type 2 diabetes in Asians: a systematic review and meta-analysis. PLoS One. 2012;7(9):e44525.

48. Wu JH, Micha R, Imamura F, Pan A, Biggs ML, Ajaz $\mathrm{O}$, Djousse L, et al. Omega-3 fatty acids and incident type 2 diabetes: a systematic review and meta-analysis. Br J Nutr. 2012;107(Suppl 2):S214-227.

49. Xiao C, Lewis GF. Regulation of chylomicron production in humans. Biochim Biophys Acta. 2012;1821(5):736746.

50. Davidson MH. Mechanisms for the hypotriglyceridemic effect of marine omega-3 fatty acids. Am J Cardiol. 2006;98(4A):27i-33i.

51. Nikkila EA, Taskinen MR, Kekki M. Relation of plasma high-density lipoprotein cholesterol to lipoprotein-lipase activity in adipose tissue and skeletal muscle of man. Atherosclerosis. 1978;29(4):497-501.

52. Morgan NG, Dhayal S. G-protein coupled receptors mediating long chain fatty acid signalling in the pancreatic beta-cell. Biochem Pharmacol. 2009;78(12):1419-1427.

53. Oh DY, Talukdar S, Bae EJ, Imamura T, Morinaga H, Fan $\mathrm{W}, \mathrm{Li}$ P, et al. GPR120 is an omega-3 fatty acid receptor mediating potent anti-inflammatory and insulin-sensitiz- ing effects. Cell. 2010;142(5):687-698.

54. Sztalryd C, Kraemer FB. Regulation of hormone-sensitive lipase in streptozotocin-induced diabetic rats. Metabolism. 1995;44(11):1391-1396.

55. Morishita M, Tanaka T, Shida T, Takayama K. Usefulness of colon targeted DHA and EPA as novel diabetes medications that promote intrinsic GLP-1 secretion. J Control Release. 2008;132(2):99-104.

56. Yanai H. Which Is Better to Prevent Cardiovascular Events, Only EPA or the Combination of EPA and DHA? J Endocrinol Metab. 2016;6(4):101-103.

57. Yokoyama M, Origasa H, Matsuzaki M, Matsuzawa Y, Saito Y, Ishikawa Y, Oikawa S, et al. Effects of eicosapentaenoic acid on major coronary events in hypercholesterolaemic patients (JELIS): a randomised open-label, blinded endpoint analysis. Lancet. 2007;369(9567):10901098.

58. Gruppo Italiano per lo Studio della Sopravvivenza nell'Infarto miocardico. Dietary supplementation with $\mathrm{n}-3$ polyunsaturated fatty acids and vitamin E after myocardial infarction: results of the GISSI-Prevenzione trial. Lancet. 1999;354(9177):447-455.

59. Tavazzi L, Maggioni AP, Marchioli R, Barlera S, Franzosi MG, Latini R, Lucci D, et al. Effect of n-3 polyunsaturated fatty acids in patients with chronic heart failure (the GISSI-HF trial): a randomised, double-blind, placebo-controlled trial. Lancet. 2008;372(9645):1223-1230.

60. Rauch B, Schiele R, Schneider S, Diller F, Victor N, Gohlke H, Gottwik M, et al. OMEGA, a randomized, placebo-controlled trial to test the effect of highly purified omega-3 fatty acids on top of modern guidelineadjusted therapy after myocardial infarction. Circulation. 2010;122(21):2152-2159.

61. Bosch J, Gerstein HC, Dagenais GR, Diaz R, Dyal L, Jung $\mathrm{H}$, Maggiono AP, et al. n-3 fatty acids and cardiovascular outcomes in patients with dysglycemia. N Engl J Med. 2012;367(4):309-318. 